\title{
Acoustic Measurements in a Tissue Mimicking Liquid
}

\author{
Michael C. Macdonald, PhD, Ernest L. Madsen, PhD
}

A liquid has been developed that mimics soft tissue in terms of propagation speed, attenuation, and nonlinearity parameter $B / A$. Since the slope of the attenuation coefficient is nearly constant up to at least $18 \mathrm{MHz}$ and the value can be anywhere in the range 0.1 through $0.7 \mathrm{~dB} /(\mathrm{cm} \cdot \mathrm{MHz})$, the material is ideal for assessing the effectiveness of attenuation derating of output acoustic intensities and pressures measured in water.

A clinical ultrasound system was used to transmit into the tissue mimicking liquid. The pulse intensity integral and rarefactional pressure at various field locations in the tissue mimicking liquid were computed and compared to the corresponding values measured in water with attenuation derating applied. The slope of the attenuation coefficient in the tissue mimicking liquid was used for the derating. From this data it is seen that the present practice of modeling in vivo exposure using linearly derated measurements made in water is not always conservative. KEY WORDS: Ultrasound; Tissue mimicking liquid; Nonlinearity.
I $\mathrm{n}$ an effort to limit patient exposure to ultrasound, the Food and Drug Administration and the AIUM have made recommendations to ultrasound equipment manufacturers that values of two parameters, which are relevant to potential bio-

\section{ABBREVIATIONS}

AIUM, American Institute of Ultrasound in Medicine; TI, Thermal index; MI, Mechanical index; NEMA, National Electrical Manufacturers' Association; PII, Pulse intensity integral; TM, Tissue mimicking; IEC, International Electrotechnical Commission; GPIB, General purpose interface bus; IEEE, Institute of Electrical and Electronics Engineers; NIST, National Institute of Standards and Technology

Received from the Acuson Corporation, Mountain View, California (M.C.M.), and the Department of Medical Physics, University of Wisconsin-Madison, Madison, Wisconsin (E.L.M.).

Address correspondence and reprint requests to Michael C. Macdonald, General Electric Medical Systems, P.O. Box 414, EA-54, Milwaukee, WI 53201. logical effects, be available to clinical users. One parameter is the TI, whose value is a predictor for temperature rise of tissue in an ultrasound beam. The other recognized potential mechanism of biological damage involves cavitation, and the likelihood of patient injury due to cavitation is thought to increase with the value of the parameter referred to as the MI. The definitions and models for the TI and MI are detailed in a standard produced by a joint committee of the AIUM and NEMA. ${ }^{1}$

Typically in acoustic output quantification, a hydrophone is used in a water tank to record the temporal acoustic pressure of a propagating wave. For the purposes here, we are interested in the most extreme rarefactional pressure $p_{r}$, used for calculating the MI, and the PII, used for calculating the temporal average intensity. The MI and the temporal average intensity are subject to restrictions imposed by the Food and Drug Administration. The temporal average intensity is also used in calculating the TI. 
The PII is given by

$$
\mathrm{PII}=\int_{T 1}^{T 2} \frac{p^{2}(t)}{\rho c} d t
$$

where $p(t)$ is the instantaneous acoustic pressure and $\rho$ and $c$ are the density and propagation speed, respectively, of the medium. The integration limits are specified by the period of time for which the hydrophone signal is nonzero.

According to the presently applicable standards, the water measured pressures and intensities are made to model the attenuative effects of tissue through the application of a derating factor. Derating is applied to any waveform by multiplying all acoustic pressures $p(t)$ (at time $t$ ) by a factor $e^{-\alpha f_{0} z}$ where $\alpha=0.3 \mathrm{~dB} /(\mathrm{cm} \cdot \mathrm{MHz})=0.0345$ neper $/(\mathrm{cm} \bullet$ $\mathrm{MHz}), f_{0}$ is the center frequency of the pulse, and $z$ is the distance from the transducer to the receiver.

Use of the deration process reasonably assumes that the propagation of the sound pulse in water or in tissue is linear. However, it is well known that most diagnostic ultrasound systems are capable of emitting pulses which are nonlinear for propagation in both water and tissue. The generation of nonlinearities in the water is a concern because, as the local intensities increase, shock formation occurs in the water and field intensities become less dependent on the source intensity. ${ }^{2}$ It is not known to what extent such nonlinearities occur in tissue, although it is thought that most tissues are less subject to such effects than water.

Models have been developed for predicting clinically relevant acoustic fields in tissuelike media.3.,4 However, experimental data are lacking with which to test these theoretical models or to test the validity of using linearly derated water measured acoustic fields to estimate acoustic exposure in tissue.

A liquid medium has been developed at the University of Wisconsin-Madison that mimics soft tissue for all relevant acoustic properties, including the nonlinearity parameter $B / A .5$ An acoustic hydrophone can be submersed in this medium for mapping the acoustic fields of transducers.

In the work presented here, two linear array transducer assemblies on the Acuson Sequoia ultrasound system (Acuson, Mountain View, CA) were used to generate acoustic fields in both water and the TM liquid. Axial peak rarefactional pressures and PIIs were determined for identical transmit conditions using both media, thus allowing a direct comparison of the linearly derated water values with the corresponding values in the TM liquid.

\section{MATERIALS}

The composition of the TM liquid used in these experiments is shown in Table 1. A more detailed description of this type of material is given in Madsen and coworkers. ${ }^{5}$

The speed of sound and the attenuation slope of the liquid were measured using a previously published through-transmission technique. ${ }^{6}$ At room temperature $\left(22^{\circ} \mathrm{C}\right)$, at which all hydrophone measurements were carried out, the speed of sound of the TM liquid was found to be $1526 \pm 1 \mathrm{~m} / \mathrm{s}$.

The attenuation coefficient slope of the liquid can be adjusted to be anywhere from 0.1 to $0.7 \mathrm{~dB} /(\mathrm{cm}$ - MHz). The liquid used in these tests was targeted to have an attenuation slope of $0.3 \mathrm{~dB} /(\mathrm{cm} \cdot \mathrm{MHz})$ at low frequencies (below $4 \mathrm{MHz}$ ). This value corresponds to the AIUM/NEMA recommended linear derating factor. The attenuation slope for a sample of the TM liquid was measured at room temperature at these lower frequencies to be about $0.29 \mathrm{~dB} /(\mathrm{cm} \cdot \mathrm{MHz})$. Over the frequency range from 2.25 to $18 \mathrm{MHz}$, however, the average attenuation slope was $0.27 \mathrm{~dB} /(\mathrm{cm} \cdot \mathrm{MHz})$. Note that the formulation can be adjusted to yield an average attenuation slope closer to $0.3 \mathrm{~dB} /(\mathrm{cm} \cdot \mathrm{MHz})$ over the broad frequency range. To assure meaningful comparisons of TM liquid and water derated values, all water measured fields reported here have been derated using the attenuation coefficient slope of $0.27 \mathrm{~dB} /(\mathrm{cm} \cdot \mathrm{MHz})$ instead of the canonical 0.3 $\mathrm{dB} /(\mathrm{cm} \cdot \mathrm{MHz}){ }^{*}$ The attenuation coefficient for the TM liquid as a function of frequency is shown in Figure 1.

A very critical property of any medium used for the study of acoustic nonlinearity is the nonlinearity parameter $B / A$. This parameter determines the rate at which the initial acoustic pulse loses energy from the fundamental frequency to higher harmonics. A $B / A$ value of 5.7 was measured for a sample of the TM liquid at room temperature using an ultrasound through-transmission method.7,8 This is on the low end of reported values for the nonlinearity parameter for tissues at $26^{\circ} \mathrm{C}$, which range from 6.3 (porcine kidney) through 6.9 (porcine liver).

The acoustic properties of the liquid used in this study are summarized in Table 2 . It is important to note that the acoustic properties of the liquid have been observed to be stable for more than 2 years. ${ }^{5}$

\footnotetext{
*Almost all data had been acquired before it was realized that the average attenuation coefficient slope was 0.27 instead of 0.29 or $0.30 \mathrm{db} /(\mathrm{cm} \cdot \mathrm{MHz})$; it was considered that 0.27 was close enough that acquisition of new data with 0.30 liquid was not necessary.
} 
Table 1: Composition of the TM Liquid*

\begin{tabular}{lc}
\hline Material & \% by Volume \\
\hline Deionized water & 60.7 \\
Evaporated milk & 36.3 \\
$n$-propanol & 3.0 \\
\hline
\end{tabular}

*Thimerosal is added as a preservative at $1 \mathrm{~g} /$ liter.

For the purposes of this study, about 40 liters of the TM liquid was prepared at the University of Wisconsin-Madison and shipped to Acuson Corporation, where it was used to fill a small automated beam plot tank.

\section{METHOD AND PROCEDURES}

Figure 2 shows the essential components of the data collection apparatus. The ultrasonic transmitter was an Acuson Sequoia system with the scanning arrested so that only pulses corresponding to the central scan line were produced. The transducers used in all cases were linear arrays, and the default $0 \mathrm{~dB}$ power level was employed in every case except when studying the nonlinear effects as a function of output power. The transducer assembly was coupled directly to the transport medium, which was either water at $37^{\circ} \mathrm{C}$ or the $\mathrm{TM}$ liquid at $22^{\circ} \mathrm{C}$. The involved pulses were interrogated with a calibrated $0.6 \mathrm{~mm}$ bilaminar shielded Marconi membrane hydrophone

Figure 1 Attenuation coefficient for the TM liquid at room temperature $\left(22^{\circ} \mathrm{C}\right)$.

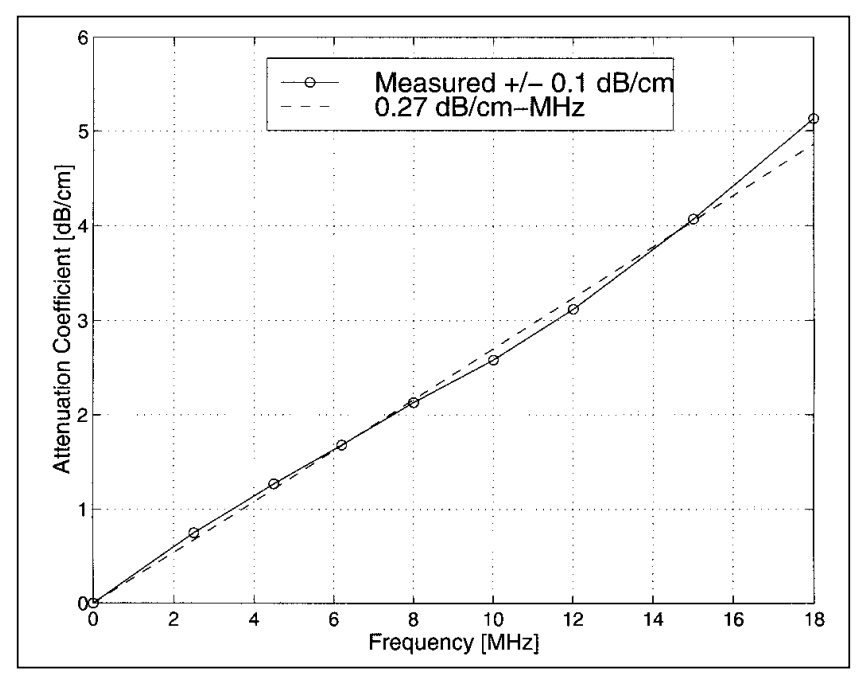

(GEC-Marconi Research Centre, Chelmsford, England). The hydrophone signals produced in water or the TM liquid were amplified and sent to a Tektronix RTD710A $200 \mathrm{MHz}$ digitizer (Tektronix, Beaverton, OR). The digitizer, under control of a UNIX workstation, then sent the digitized waveform to a storage device on the workstation via a GPIB IEEE-488 interface.

The hydrophone was calibrated at discrete operating frequencies and at very low output power (assuring minimal nonlinear effects) in both liquid media using a set of narrow band NIST traceable source transducers, which have known ultrasonic power output at various center frequencies. A planar scan at the focal distance was done in each media to determine a frequency dependent calibration. The attenuation in the TM liquid, of course, had to be taken into account.

The hydrophone was positioned at various locations in the acoustic field of the transducer using a custom-built three-axis micropositioner. Each axis of the positioner was under the control of the UNIX workstation via a Unidex motion controller (Aerotech, Pittsburgh, PA) and had a spatial resolution of about $20 \mu \mathrm{m}$. The transducer under test was aligned in the tank such that the location of peak PIIs at two axial depths was colinear with the movement of the hydrophone along the $\mathrm{Z}$ (depth) axis of the positioner. This was done to ensure that the center of the ultrasound beam could be interrogated by moving the hydrophone with the $\mathrm{Z}$ axis positioner.

For all data presented here, the hydrophone was translated along the beam axis and waveforms recorded for various depths and transmit power settings. The values of $p_{r}$ and PII were computed for

Figure 2 Diagram of the data acquisition system.

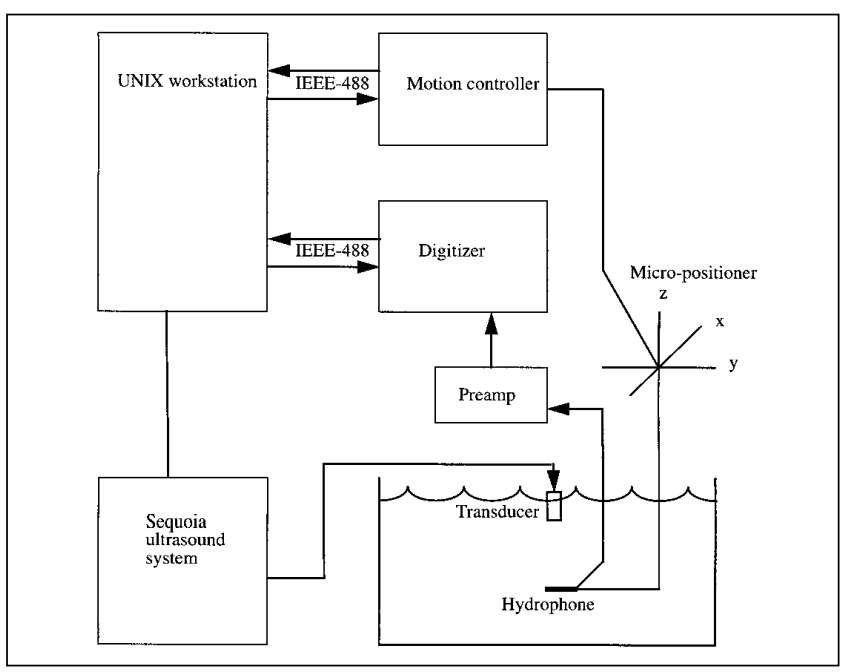


each waveform using $\mathrm{C}++$ routines on the workstation. Of particular interest here were the differences in $p_{r}$ and PII as measured in the TM liquid and in water with derating. A derating factor of $0.27 \mathrm{~dB} /$ $(\mathrm{cm} \cdot \mathrm{MHz})$ was applied to all waveforms measured in water. This derating factor was chosen instead of the AIUM/NEMA standard of $0.3 \mathrm{~dB} /(\mathrm{cm} \cdot \mathrm{MHz})$ to equal the average attenuation coefficient slope of the TM liquid.

\section{RESULTS}

Figure 3 shows the PII at discrete points along the beam axis of a linear array transducer with a nominal frequency of $5 \mathrm{MHz}$ and a focal depth of $2 \mathrm{~cm}$. The PII as measured in the TM liquid and in water are shown with the water measured values derated using $0.27 \mathrm{~dB} /(\mathrm{cm} \cdot \mathrm{MHz})$.

Figure 4 is for the same operating condition as Figure 3, only now the $p_{r}$ is shown as a function of depth for propagation into the TM liquid and for propagation in water, with a $0.27 \mathrm{~dB} /(\mathrm{cm} \cdot \mathrm{MHz})$ derating applied to the water data. The operating condition used for the data in Figures 3 and 4 does not represent very aggressive focusing. That is, the ratio of the beam cross-sectional area at the transducer face to the beam cross-sectional area at the measurement depth is not as high as is commonly possible.

Figure 5 is another plot showing the PII at points

Figure 3 Comparison of PII in the TM liquid and water along the beam axis of a linear array transducer with a $5 \mathrm{MHz}$ nominal frequency and transmit depth of $2.0 \mathrm{~cm}$. The water measured PII values were derated using a $0.27 \mathrm{~dB} /(\mathrm{cm} \cdot \mathrm{MHz})$ derating factor.

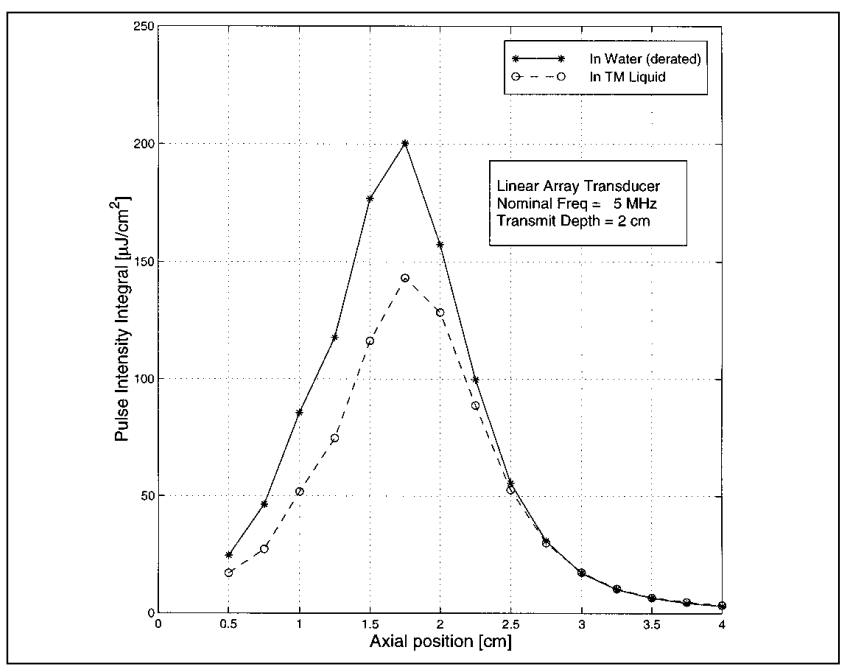

along the beam axis of a linear array transducer. The nominal frequency was again $5 \mathrm{MHz}$, but the focal depth was $7 \mathrm{~cm}$. Again, the PII as a function of axial depth is shown for propagation in the TM liquid and for propagation in water with a $0.27 \mathrm{~dB} /(\mathrm{cm} \cdot \mathrm{MHz})$ derating applied to the water data.

Figure 6 is for the same operating condition as in Figure 5 and shows $p_{r}$ as a function of depth for propagation in the TM liquid and for propagation in water with a $0.27 \mathrm{~dB} /(\mathrm{cm} \cdot \mathrm{MHz})$ derating applied to the water data. Unlike the operating condition used for the data in Figures 3 and 4, the operating condition for Figures 5 and 6 had fairly aggressive focusing.

Figures 7 through 10 show the change in $p_{r}$ and PII as the transmit power is varied. Each figure shows data for a discrete field location and operating condition.

Figures 7 and 8 show the PII and $p_{r}$, respectively, for a nominal frequency of $5 \mathrm{MHz}$ and a transmit depth of $2.0 \mathrm{~cm}$. The PII and $p_{r}$ are shown as measured in the TM liquid and in water with a $0.27 \mathrm{~dB} /$ $(\mathrm{cm} \cdot \mathrm{MHz})$ derating applied. The measurement depth was $1.8 \mathrm{~cm}$ from the transducer face along the beam axis, and the transmit power from the transducer was varied from $0 \mathrm{~dB}$ (full output power) to $-21 \mathrm{~dB}$, corresponding to an $\mathrm{MI}$ range from a maximum of 0.5 down to 0.1 .

Figures 9 and 10 show PII and $p_{r}$ as a function of transmit power as measured in the TM liquid and water with a $0.27 \mathrm{~dB} /(\mathrm{cm} \cdot \mathrm{MHz})$ derating for a nom-
Figure 4 Comparison of peak rarefactional pressure in the TM liquid and water along the beam axis of a linear array transducer with a $5 \mathrm{MHz}$ nominal frequency and transmit depth of 2.0. $\mathrm{cm}$. The water measured $p_{r}$ values were derated using a $0.27 \mathrm{~dB} /(\mathrm{cm} \cdot \mathrm{MHz})$ derating factor.

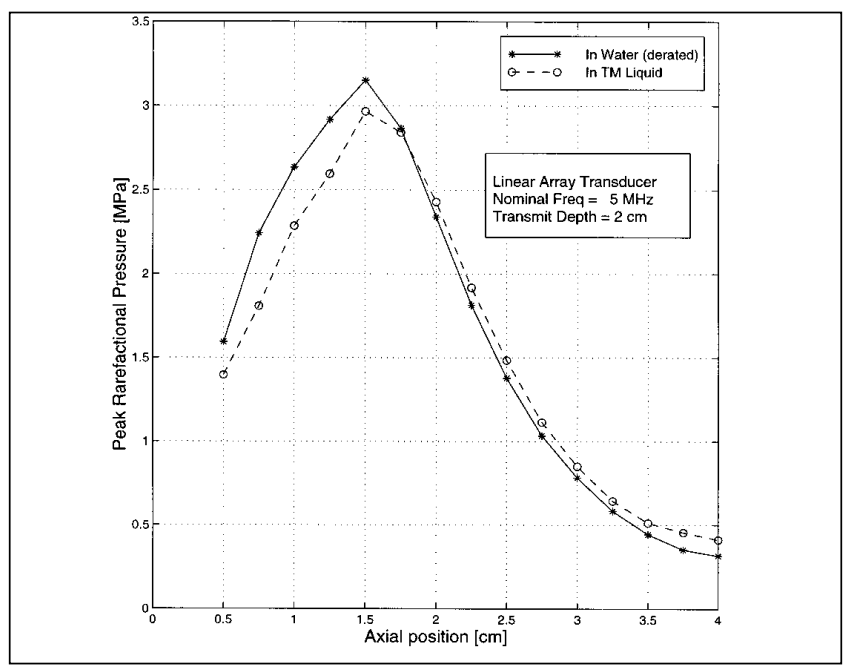


inal frequency of $5 \mathrm{MHz}$ and transmit depth of $7 \mathrm{~cm}$. The measurement location was along the beam axis, $6.8 \mathrm{~cm}$ from the transducer face. Here the computed MI ranges from 0.4 down to 0.04 .

\section{DISCUSSION}

From the previously presented figures, it is clear that the derated water intensities and pressures are not necessarily conservative with respect to the values measured in the TM liquid.

In Figures 3 and 4, both the PII and $p_{r}$ in the TM liquid are almost always conservatively estimated using the derated water values. Only beyond the axial depth of the $2 \mathrm{~cm}$ transmit focus does the value of $p_{r}$ in the TM fluid become underestimated by the derated water value, as seen in Figure 4.

A similar axial sampling of PII and $p_{r}$ for another linear array and another operating condition is shown in Figures 5 and 6. Here, however, clearly both the PII and $p_{r}$ in the TM liquid are underestimated at almost all axial depths by the derated water values. Note that the operating condition used for obtaining the data in Figures 5 and 6 exhibited much higher focal gain than was present for the operating condition associated with Figures 3 and 4, and the transmit focus was $7 \mathrm{~cm}$ instead of $2 \mathrm{~cm}$.

Figures 7 through 9 are helpful in identifying to what extent transmit power affects the generation of nonlinearities. At very low transmit powers it is

Figure 5 Comparison of PII in the TM liquid and water along the beam axis of a linear array transducer with a $5 \mathrm{MHz}$ nominal frequency and transmit depth of $7.0 \mathrm{~cm}$. The water measured PII values were derated using a $0.27 \mathrm{~dB} /(\mathrm{cm} \cdot \mathrm{MHz})$ derating factor.

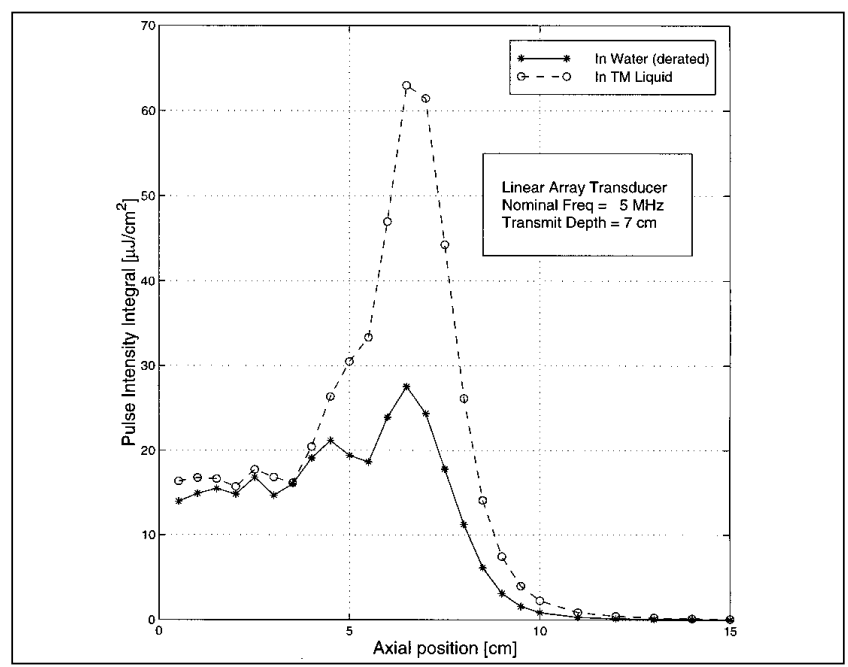

expected that the propagating waveforms are approximately linear. If that is true, then the derated pressures and intensities as measured in water should be approximately equal to the values measured in the TM liquid.

In Figure 7, for instance, it is clear that the derated water PII at all transmit powers overestimates the PII found when measured in the TM liquid. The PII values, as measured in both water and the TM liquid, increase fairly linearly with increasing transmit power, except that the water measured PII starts to exhibit a nonlinear behavior at the very highest transmit power. Note that the change in PII as measured in the TM fluid is approximately linear through the entire range of transmit powers. It is probably safe to assume that, for transmit powers higher than those available here, the derated water value for PII would have fallen below the PII as measured in the TM liquid.

Figure 8, which shows the $p_{r}$ data as a function of the transmit power for the same operating condition as in Figure 7, includes a "cross-over" of the derated water measured $p_{r}$ and the $p_{r}$ as measured in the TM liquid. Note that for the $0 \mathrm{~dB}$ transmit power case, the TM liquid value for $p_{r}$ is slightly higher than that computed by derating the $p_{r}$ as measured in water.

Figure 9 shows how the generation of nonlinearities in water can affect the validity of using a linear attenuation model for derating. At lower transmit powers the derated water values for PII are approximately equal to, but slightly higher than, the PII

Figure 6 Comparison of peak rarefactional pressure in the TM liquid and water along the beam axis of a linear array transducer with a $5 \mathrm{MHz}$ nominal frequency and transmit depth of $7.0 \mathrm{~cm}$. The water measured $p_{r}$ values were derated using a $0.27 \mathrm{~dB} /(\mathrm{cm} \bullet \mathrm{MHz})$ derating factor.

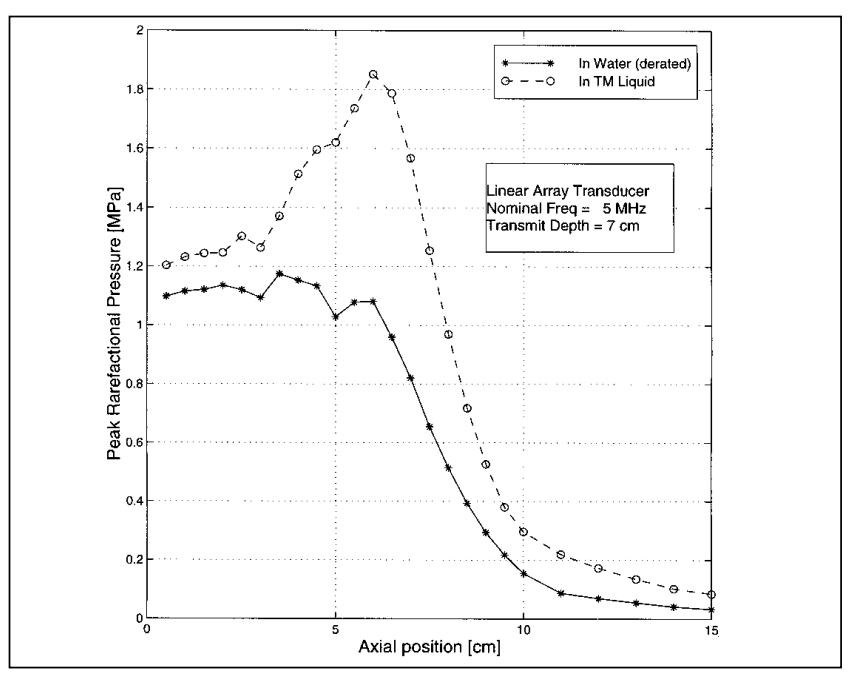




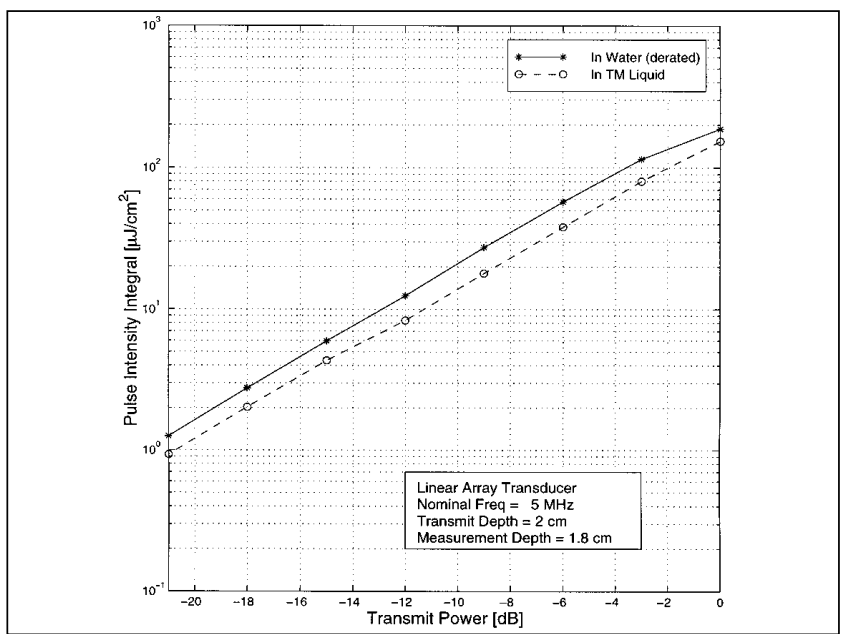

Figure $7 \mathrm{PII}$ in the TM liquid and in water, with $0.27 \mathrm{~dB}$ / $(\mathrm{cm} \cdot \mathrm{MHz})$ derating factor applied, as a function of transmit power from the transducer assembly. The intensity is measured at a depth of $1.8 \mathrm{~cm}$ from the linear array transducer, and the operating condition had a nominal operating frequency of $5 \mathrm{MHz}$ and a transmit depth of $2.0 \mathrm{~cm}$.

measured in the TM fluid. As the transmit power is increased, however, the derated PII in water begins to underestimate the PII measured in the TM fluid. Here the nonlinear behavior is quite apparent.

Figure $9 \mathrm{PII}$ in the TM liquid and in water, with $0.27 \mathrm{~dB}$ / $(\mathrm{cm} \cdot \mathrm{MHz})$ derating factor applied, as a function of transmit power from the transducer assembly. Here the intensity is measured $6.8 \mathrm{~cm}$ from the linear array transducer along the beam axis, and the operating condition had a nominal operating frequency of $5 \mathrm{MHz}$ and a transmit depth of $7.0 \mathrm{~cm}$.

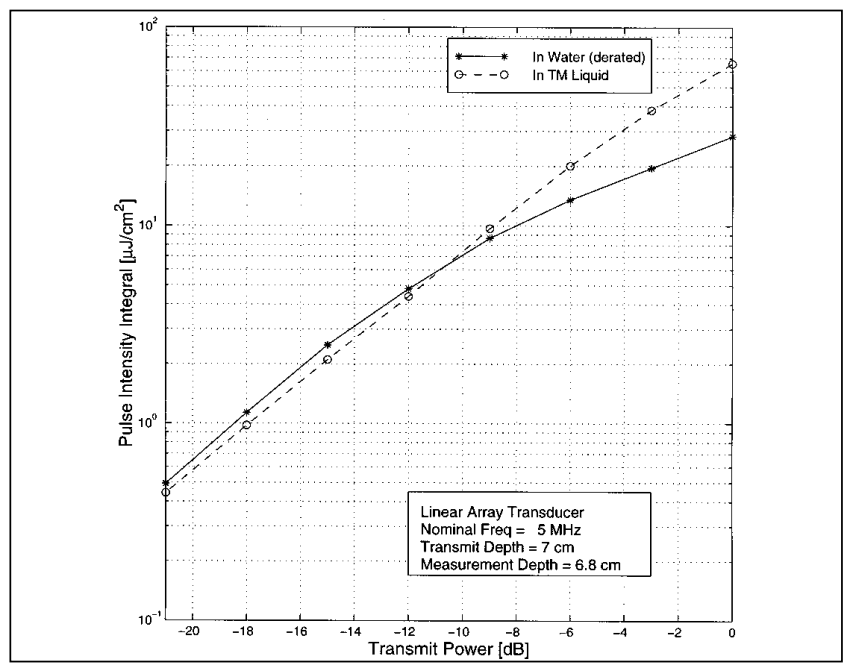

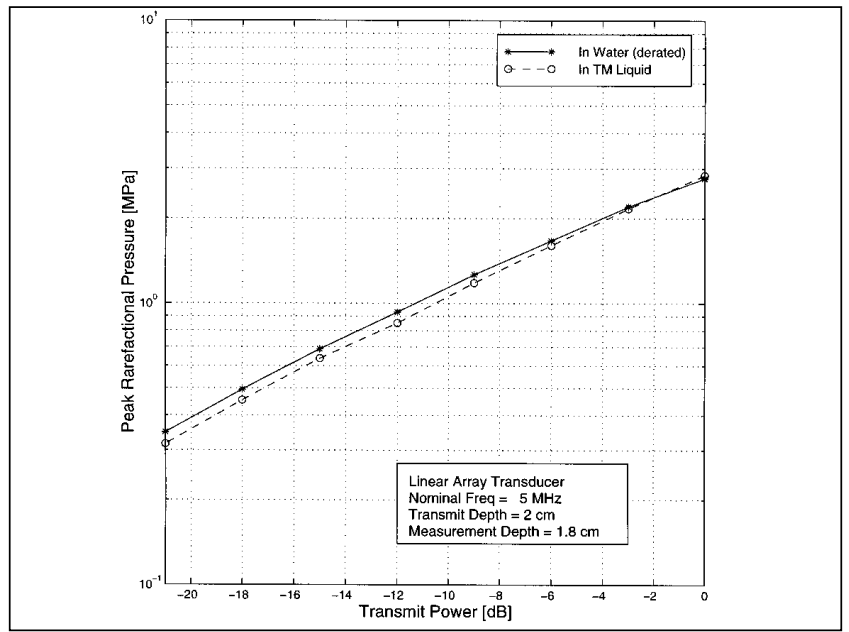

Figure $8 p_{r}$ in the TM liquid and in water, with $0.27 \mathrm{~dB}$ / $(\mathrm{cm} \cdot \mathrm{MHz})$ derating factor applied, as a function of transmit power from the transducer assembly. The values were measured at a depth of $1.8 \mathrm{~cm}$ from the linear array transducer, and the operating condition had a nominal operating frequency of $5 \mathrm{MHz}$ and a transmit depth of $2.0 \mathrm{~cm}$.

Figure 10 shows the divergence of $p_{r}$ versus power as power increases for the TM liquid case and water derated case.

It is worth noting that the PII as measured in the TM fluid also begins to exhibit a nonlinear behavior at the highest transmit powers. This indicates that nonlinear distortion of the waveforms in the TM

Figure $\mathbf{1 0} p_{r}$ in the TM liquid and in water, with $0.27 \mathrm{~dB} /(\mathrm{cm}$ - $\mathrm{MHz}$ ) derating factor applied, as a function of transmit power from the transducer assembly. The values were measured at a depth of $6.8 \mathrm{~cm}$ from the linear array transducer, and the operating condition had a nominal operating frequency of $5 \mathrm{MHz}$ and a transmit depth of $7.0 \mathrm{~cm}$.

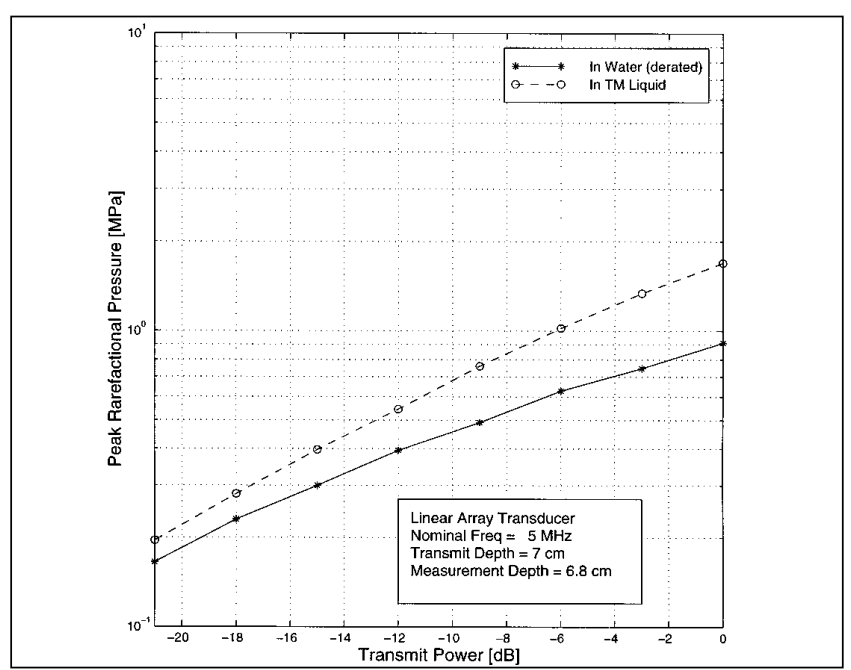


fluid was significant and may need to be accounted for in any mathematical models for waveform propagation through tissue.

It should be noted that over a period of 12 months, during which the hydrophone was immersed in the TM liquid about $12 \mathrm{~h}$ per month, the normally visible "luster" of the hydrophone gold electrodes was somewhat diminished. This could possibly correspond to a degradation of the electrodes. If a degradation of the hydrophone is occurring, two components of the TM liquid are suspect: $n$-propanol and thimerosal. The thimerosal has also been found to cause "bleeding" of the black dye from black silicone rubber seals over a period of a few months. The $n$-propanol, which is present to elevate the ultrasonic propagation speed, can be replaced for that purpose with glycerol, a much less chemically active material. In fact, glycerol is superior to $n$-propanol because it does not evaporate even in an open bath, leaving water the only volatile material in the TM material, and water can be replenished easily. If thimerosal is causing electrode degradation, then other preservatives can be tried. An alternative to thimerosal as a preservative, 1-(cis-3-chloroallyl)-3,5,7-triaza-1azonia-adamantane chloride, had been tested for 3 months at the time of this writing, with no change in ultrasonic properties or appearance of the material and no bleeding of black color from silicone rubber seals.

The question has been raised regarding long-term stability of the liquid TM material relative to the possibility that it might be included as a basis for an IEC standard. The ultrasonic properties of propagation speed and attenuation coefficients were monitored throughout the study reported in this article, and they were stable. The material was kept in a closed container when not in use, but it was used intermittently over a rather long period of time (about 12 months). Long-term care of the material is required, however: storing it in a closed container when not in use and stirring it to assure uniformity if it has been unused for a week or more. Glycerol should be used for controlling (raising) propagation speed instead of $n$-propanol, and, if the alternative preservative proves adequate, it should be used instead of thimerosal. If the glycerol and alternative preservative form of the material passes long-term stability tests, then that would seem to be an adequate material for an IEC standard.

The liquid holding tank and other apparatus used in this study were adapted from systems employing water, where care of the liquid transmission medium is not a significant concern. It is likely that a system specifically designed for use with a smaller volume of TM liquid could be designed. For instance, it may be helpful to employ an upward-directed hydrophone, which can be translated vertically, with a source directed downward and subject to three-dimensional translations (and perhaps rotations) using apparatus external to the TM liquid. A flexible plastic sheet with an opening for the source transducer could prevent evaporation. Such a tank could conceivably require as little as 20 liters of TM liquid.

\section{SUMMARY AND CONCLUSIONS}

The current method for determining the PII and peak rarefaction acoustic pressure on a $0.3 \mathrm{~dB} /(\mathrm{cm} \cdot \mathrm{MHz})$ medium is to make measurements in degassed water and then "derate" the values by multiplying acoustic pressures $p(t)$ at times $t$ by a factor $e^{-\alpha f_{0} z}$ where $\alpha=$ $0.3 \mathrm{~dB} /(\mathrm{cm} \cdot \mathrm{MHz})=0.0345$ neper $/(\mathrm{cm} \cdot \mathrm{MHz}), f_{0}$ is the center frequency of the pulse, and $z$ is the distance from the transducer to the receiver. The resulting PII and $p_{r}$ are used in determining acoustic output display parameters MI and TI. The accuracy of this derating scheme was tested to determine whether serious errors were made due to ignoring nonlinear propagation effects. Note that the derating model also ignores beam hardening (shift to more penetrating lower frequencies) due to frequency dependent attenuation.

Tests were done using clinical transducers with $5 \mathrm{MHz}$ nominal frequency. Pulses were transmitted into water and TM liquid under identical conditions and derated results compared with those in the TM liquid. For short transmission depths ( $2 \mathrm{~cm}$ focus) the water derated PII and $p_{r}$ were slightly greater than those found using the TM liquid, an acceptable safety condition. However, when the transmit depth was greater $(7 \mathrm{~cm}$ focus) and nonlinear propagation effects presumably larger, the maximum axial PII in the TM liquid was more than twice that in water with derating, and the peak value of $p_{r}$ was about $80 \%$ higher in the TM liquid than in water with derating.

These results indicate that derated values of PII and $p_{r}$ determined in water are not necessarily conservative, and a more appropriate means of predicting in vivo ultrasonic exposure is needed. This may be accomplished by (1) developing better models for derating the water measured pressures, (2) using the conventional measurement protocols, but with the TM liquid instead of water, or (3) using

Table 2: Acoustic Properties of the TM Liquid at Room Temperature

$\begin{array}{lc}\text { Speed of sound }(\mathrm{m} / \mathrm{s}) & 1526 \\ \text { Density }(\mathrm{g} / \mathrm{cc}) & 1.03 \\ \text { Attenuation coefficient slope }[\mathrm{dB} /(\mathrm{cm} \cdot \mathrm{MHz})] & 0.27 \\ \text { Nonlinearity coefficient } B / A & 5.7\end{array}$


mathematical models for finite amplitude sound beams, for which the TM liquid may serve as a valuable transmission medium for the validation of the models.

\section{REFERENCES}

1. AIUM/NEMA: Standard for Real-Time Display of Thermal and Mechanical Indices on Diagnostic Ultrasound Equipment. Rockville, MD, American Institute of Ultrasound in Medicine and National Electrical Manufacturers' Association, 1992

2. Muir TG, Carstensen EL: Prediction of nonlinear acoustic effects at biomedical frequencies and intensities. Ultrasound Med Biol 6:345, 1980

3. Reilly CR, Parker KJ: Finite-amplitude effects on ultrasound beam patterns in attenuating media. J Acoust Soc Am 86:2339, 1989

4. Christopher T, Carstensen EL: Finite amplitude distortion and its relationship to linear derating formulae for diagnostic ultrasound systems. Ultrasound Med Biol 22:1103, 1996
5. Madsen EL, Frank GF, Dong F: Liquid or solid ultrasonically tissue-mimicking materials with very low scatter. Ultrasound Med Biol 4:24:535, 1998

6. Madsen EL, Zagzebski JA, Frank GR: Oil-in-gelatin dispersions for use as ultrasonically tissue-mimicking materials. Ultrasound Med Biol 8:277, 1982

7. Gong XF, Zhu ZM, Shi T, et al: Determination of the acoustic nonlinearity parameter in biological media using FAIS and ITD methods. J Acoust Soc Am 86:1, 1989

8. Dong F, Macdonald MC, Madsen EL, et al: Nonlinearity parameter B/A of tissue-mimicking materials with very low scatter, Program and Abstracts, 23rd International Symposium on Ultrasonic Imaging and Tissue Characterization, May 1998, Arlington, VA 